\title{
Alterações Metabólicas do Paciente Infectado por HIV
}

$\mathrm{O}$ TRATAMENTO ANTI-RETROVIRAL contemporâneo (TARV), baseado em esquemas contendo pelo menos três drogas, mostrou-se altamente eficaz na redução da morbi-mortalidade associada à infecção pelo vírus da imunodeficiência humana (HIV). Entretanto, o TARV pode induzir complicações metabólicas graves, tais como resistência à insulina (RI), síndrome metabólica (SM), lipodistrofia (LDF) e doenças cardiovasculares (DCV). Os efeitos metabólicos do TARV no incremento do risco de aterosclerose precoce e acelerada, em pacientes infectados por HIV, são bem reconhecidos. Essas condições clínicas inter-relacionadas têm prevalência significativamente maior entre pacientes infectados por HIV em uso de TARV.

Alterações do metabolismo da glicose em graus variados têm sido associadas ao TARV. Glicemias entre 140-199 mg/dL, medidas após duas horas da ingesta oral de $75 \mathrm{~g}$ de glicose (TOTG), ou glicemias de jejum entre 100-125 mg/dL sugerem a presença de RI. Quiçá o método mais prático seja, no entanto, através do Homeostasis Model Assessment HOMA-IR, calculado como [insulina de jejum $(\mathrm{mU} / \mathrm{mL})$ x glicemia de jejum $(\mathrm{mmol} / \mathrm{L})] / 22,5$. Em bons laboratórios, esse método tem excelente correlação com o clamp hiperinsulinêmico euglicêmico, considerado o padrão-ouro da determinação de IR. Não há consenso acerca do valor de HOMA acima do qual se diagnostica resistência à insulina, variando de 2,5 a 3,5. Curiosamente, apesar de a população a partir da qual se calcula o HOMA "normal" dever ter tolerância normal à glicose e IMC $<25$ $\mathrm{kg} / \mathrm{m}^{2}$, alguns laboratórios corrigem os valores de HOMA para o IMC do paciente. Tal prática subestima grosseiramente a prevalência de RI.

São bem reconhecidos que obesidade, idade superior a 45 anos e história familiar de diabetes mellitus (DM) são fatores de risco para RI. No paciente infectado por HIV usando TARV, somam-se outros fatores: lipodistrofia, uso de inibidores de protease (IP) e co-infecção pelo vírus da hepatite $\mathrm{C}(\mathrm{HCV})$. Apesar de a maior parte dos pacientes manter glicemia de jejum normal durante TARV, até $40 \%$ dos pacientes com lipodistrofia apresentarão intolerância à glicose, diagnosticada por TOTG. Estudo do Multicenter AIDS Cohort (MACS) (1) revelou prevalência de DM de 14\% entre pacientes infectados recebendo TARV, comparado com $5 \%$ entre os não-infectados, após ajuste para idade e índice de massa corporal. Nos pacientes usando TARV, a incidência de DM e pré-DM foi maior naqueles que recebiam IP, estavudina e efavirenz. A presença de $\mathrm{HCV}$ aumentou 2,5 vezes o risco de DM. Outro estudo prospectivo (Data Collection on Adverse Events of Anti-HIV Drugs - DAD) (2), envolvendo 23.441 pacientes, mostrou incidência de DM de 5,9/1.000 pacientes/ano. Uso de IP contribuiu para aumento de 6\% de DM por ano de exposição.

Ainda existem inúmeras lacunas na fisiopatogenia da RI associada ao TARV. Estudos in vitro demonstram que os IP reduzem a captação de glicose mediada por insulina em musculatura esquelética e em adipócitos, interferindo no transportador transmembrana de glicose GLUT-4. Esse editorial

\section{adauto Castelo filho \\ PaUlo Abrão}

Professor Adjunto (ACF) e Médico (PA), Disciplina de Infectologia, Departamento de Medicina, Universidade Federal de São Paulo (UNIFESP), SP. 
efeito, no entanto, é muito minimizado quando o IP é o atazanavir e máximo quando o IP é ritonavir. Outro mecanismo de indução de RI por IPs é através de seu efeito no fator de transcrição SREBP-1 (steroid regulatory element binding protein-1c), afetando o metabolismo da glicose ao produzir expressões imperfeitas do receptor gama ativado por proliferador de peroxisoma (PPAR- $\gamma)$. O PPAR- $\gamma$ cumpre papel importante no metabolismo de glicose e lipídeos e agonistas do PPAR- $\gamma$, como as tiazolidinedionas (rosiglitazona, pioglitazona), melhoram a sensibilidade à insulina.

A hiperinsulinemia, associada à RI, é fator de risco reconhecido de doença coronariana em pacientes não-infectados por HIV e pode contribuir para o risco aumentado de infarto agudo do miocárdio em pacientes recebendo TARV. Portanto, intervenções para corrigir RI são claramente indicadas. Medidas não-farmacológicas se apóiam na redução de peso e aumento da atividade física. Estratégias farmacológicas utilizadas no paciente não infectado por HIV têm também sido usadas em pacientes infectados: metformina e tiazolidinedionas (rosiglitazona, pioglitazona). Apesar de ambas alternativas diminuírem a hiperinsulinemia e a glicemia, esses agentes produzem efeitos muito distintos na distribuição de gordura corpórea. Metformina promove redução no peso, no IMC, na massa total de gordura e na gordura subcutânea, enquanto as tiazolidinedionas aumentam significativamente esses parâmetros. Assim, quando predomina obesidade visceral, com aumento da circunferência abdominal, metformina é provavelmente mais indicada, sobretudo na presença de colesterol total, LDL ou triglicérides aumentados. Entretanto, esses efeitos da metformina só são observados em pacientes infectados por HIV que apresentam níveis de insulina de jejum elevados. Já as tiazolidinedionas são mais indicadas em pacientes com RI e lipoatrofia de membros, mas podem se associar com elevação de LDL. Devem ser evitadas em pacientes com ALT maior que 2,5 vezes o limite superior da normalidade.

Síndrome metabólica (SM) é um conjunto de fatores clínico-laboratoriais que identificam indivíduos sob maior risco de evento cardiovascular. Essa síndrome inclui distúrbios no metabolismo da glicose, insulina e lipídico. Após ajuste para idade, sexo, níveis de colesterol, atividade física e tabagismo, portadores de síndrome metabólica têm mortalidade por doença coronária duas a três vezes maior. A definição de síndrome metabólica do U.S. National Cholesterol Education Program Adult Treatment Panel III (ATPIII) requer presença de pelo menos três entre cinco dessas condições: obesidade abdominal (cintura $>102 \mathrm{~cm}$ homens e $80 \mathrm{~cm}$ mulheres), triglicérides $\geq 150$ $\mathrm{mg} / \mathrm{dL}$, HDL-C $<40 \mathrm{mg} / \mathrm{dL}$ nos homens e $<50$ $\mathrm{mg} / \mathrm{dL}$ nas mulheres, pressão arterial $\geq 130 / 85$ $\mathrm{mmHg}$ ou HAS em tratamento e glicemia $\geq 100$ $\mathrm{mg} / \mathrm{dL}$. Mais recentemente, a International Diabetes Federation (IDF) publicou uma classificação mais universal, que permite estudos epidemiológicos em diferentes populações. A prevalência de SM em homens HIV-positivos é significativamente maior (risco relativo 1,5 , intervalo de confiança de $95 \%, 1,14-1,98$ ) que em homens HIV-negativos. Pacientes HIV-positivos apresentam prevalência significativamente maior de níveis elevados de glicemia de jejum e triglicerídeos e níveis baixos de colesterol HDL, mas aumento da circunferência abdominal é mais prevalente em indivíduos HIV-negativos (3).

Essas complicações metabólicas tornam o paciente infectado por HIV com risco aumentado de aterosclerose e DM. O estudo Data Collection on Adverse Events from Anti-HIV Drugs (DAD), com mais de 23.000 participantes de vários países, mostrou aumento de $26 \%$ na taxa de infarto do miocárdio por ano de TARV (4). Pacientes infectados por HIV com SM têm alteração da dilatação arterial mediada por fluxo e espessamento de camada íntima-média semelhante a pacientes com DM. Evidências recentes indicam que o HIV causa inflamação arterial que certamente contribui no aumento do risco cardiovascular.

Alterações morfológicas observadas em pacientes em uso de TARV, que incluem lipoatrofia, lipoacumulação ou combinação destas condições, são usualmente referidas como lipodistrofia (LDF). Geralmente, acompanham-se de graves alterações metabólicas, como hiperglicemia, hiperinsulinemia, hiperlipidemia e hipertensão. A incidência de LDF em pacientes portadores do HIV é muito variável $(2 \%$ a $83 \%$ ), dependendo da definição utilizada. Recentemente, uma definição de caso foi proposta e validada pelo Lipodistrophy Case Definition Study Group, baseada em um estudo caso-controle, identificou 10 variáveis para compor um escore diagnóstico e de gravidade para LDF: sexo, idade, duração da infecção pelo HIV, estágio da infecção pelo HIV, relação da circunferência WAIST/HIP, ânion gap, nível sérico de colesterol HDL, gordura em membros inferiores, relação de gordura tronco/membros, relação de gordura intra-abdomina/abdominal superficial. Juntamente com exames de imagem como DEXA (dual-energy $x$ ray absorptiometry scan) ou tomografia computadorizada, obtém-se sensibilidade de $79 \%$ e especificidade de $80 \%$ para o diagnóstico de LDF. Os fatores de risco de LDF são: exposição à estavudina e IPs, idade, 
CD4 baixo, carga viral elevada, duração de TARV, raça branca e sexo feminino. Lipoatrofia associa-se com redução da diferenciação e tamanho do adipócito, apoptose do adipócito mediada por citocinas próinflamatórias (TNF- $\alpha$ ) e toxicidade mitocondrial secundária ao TARV.

Por todo o exposto acima, o tratamento adequado das alterações metabólicas do paciente infectado por HIV pressupõe parceria produtiva entre infectologista, endocrinologista e cardiologista.

\section{REFERÊNCIAS}

1. Brown TT, Li X, Cole SR, Kingsley LA, Palella F, Riddler S, et al. Cumulative exposure to nucleoside analogue reverse transcriptase inhibitors is associated with insulin resistance markers in the Multicenter AIDS Cohort Study. Arch Intern Med 2005;165:1179-84.

2. Sabin C, Friis-Moller N, Reiss P, Weber R, d'Arminio Monforte $A$, Dabis $F$, et al. Risk factors for new onset diabetes mellitus (DM) in HIV patients. Program, 3rd IAS Conference on HIV Pathogenesis and Treatment; July 24-27, 2005; Rio de Janeiro, Brazil (Abstract TuPe2.2B28).
3. Palella F, Wang Z, Chu H, Riddler S, Visscher B, Dobs A, et al. Correlates and prevalence of the metabolic syndrome over time in the Multicenter AIDS Cohort Study (MACS). Program, 3rd IAS Conference on HIV Pathogenesis and Treatment; July 24-27, 2005; Rio de Janeiro, Brazil (Abstract TuPe2.2B18).

4. Friis-Møller N, Sabin CA, Weber R, d'Arminio Monforte A, ElSadr WM, Reiss $P$, et al. and the Data Collection on Adverse Events of Anti-HIV Drugs (DAD) Study Group: Combination antiretroviral therapy and the risk of myocardial infarction. $\mathbf{N}$ Engl J Med 2003;349:1993-2003.

5. Kenneth AL. Redefining lipodistrofy syndrome. JAIDS 2005;39(4):395-400.

Endereço para correspondência:

Adauto Castelo Filho

Av. Jandira 610, apto. 32

04080-004 São Paulo, SP 